\title{
A NOTE ON HOMEOMORPHIC MEASURES ON TOPOLOGICAL GROUPS
}

\author{
by SIDNEY A. MORRIS and VINCENT C. PECK \\ (Received 20th October 1981)
}

\section{Introduction}

The classical von Neumann-Oxtoby-Ulam Theorem states the following:

Given non-atomic Borel probability measures $\mu, \lambda$ on $I^{n}$ such that

(1) $\mu(A)>0, \lambda(A)>0$ for all open $A \subset I^{n}$

(2) $\mu\left(\partial I^{n}\right)=\lambda\left(\partial I^{n}\right)=0$,

there exists a homeomorphism $h$ of $I^{n}$ onto itself fixing the boundary pointwise such that for any $\lambda$-measurable set $S$

$$
\mu(h(S))=\lambda(S)
$$

It is known that the above theorem remains valid if $I^{n}$ is replaced by any compact finite dimensional manifold [2], [4] or with $I^{\infty}$, the Hilbert cube, [8].

We shall say that a space $X$ has the homeomorphic measure property if the above theorem remains valid with $I^{n}$ replaced by $X$.

In this note we characterise those compact connected abelian metric groups having the homeomorphic measure property as precisely those which are locally connected. These are $T^{a}$, where $T$ is the circle group and $a$ is a non-negative integer or possibly $\aleph_{0}$.

Our proof depends on the following key result:

Theorem A. [6] A countable product of finite dimensional compact manifolds has the homeomorphic measure property.

\section{Compact connected metrisable abelian groups}

We shall regard $T$, the circle group, as the unit circle on the complex plane with complex multiplication as the group operation.

Lemma 1. If $f$ is a continuous homomorphism of $G \cong T^{m}$, onto $H \cong T^{n}$, there exist subgroups $A$ and $B$ of $G$ such that $G=A \times B, A \cong T^{n}$ and $B \cong T^{m-n}, f(\{e\} \times B)=\{e\}$ and $f(A \times\{e\})=H$. 
Proof. Let $C$ be the kernel of $f$. Then $C=B \times F$ where $B \cong T^{m-n}$ and $F$ is a finite group. As $B \subseteq G$ and $B \cong T^{m-n}$, Section 25.31 in [3] implies $G=A \times B$ where $A \cong T^{n}$.

Lemma 2. If $f$ is a continuous homomorphism of $G \cong T^{n}$ onto $H=H_{1} \times \cdots \times H_{n}$ where each $H_{i} \cong T$, then there exist subgroups $G_{1}, G_{2}, \ldots, G_{n}$ such that $G=G_{1} \times \cdots \times G_{n}, G_{i} \cong T$, and $f\left(G_{i}\right)=H_{i}$ for $i=1,2, \ldots, n$.

Proof. Now $f^{-1}\left(H_{1}\right)$ is a subgroup of $T^{n}$ and so is $G_{1} \times F$ where $G_{1} \cong T^{r}, r \geqq 1$, and $F$ is a finite group. But then $f\left(G_{1}\right)$ is a connected subgroup of $H_{1}$ and so $f\left(G_{1}\right)=H_{1}$. As $G_{1} \cong T^{r}$, we must have $G=B \times G_{1}$ where $B=T^{n-r}$ and $f(B)=\{e\} \times H_{2} \times \cdots \times H_{n} \cong T^{n-1}$. So $n-r \geqq n-1$, which implies $r=1$.

So $G=B_{1} \times G_{1}, G_{1} \cong T, f\left(G_{1}\right)=H_{1}$ and $f\left(B_{1}\right)=\{e\} \times H_{2} \times \cdots \times H_{n}$. As above, there is a subgroup $G_{2} \subseteq B_{1}$ such that $f\left(G_{2}\right)=H_{2}$ and $G_{2} \cong T$. Continue inductively choosing $G_{1}, \ldots, G_{n}$.

So we have subgroups $G_{1}, \ldots, G_{n}$ of $G$ each isomorphic to $T$ and $G_{i} \cap G_{j}=\{e\}$ for $i \neq j$. Thus the group generated by $G_{1}, G_{2}, \ldots, G_{n}$ is $G_{1} \times \cdots \times G_{n}$ and is isomorphic to $T^{n}$. As $G_{1} \times \cdots \times G_{n} \subseteq G \cong T^{n}$ we must have $G=G_{1} \times \cdots \times G_{n}$ and the lemma is proved.

Observe that any continuous homomorphism $f$ of $T$ into $T$ is $f\left(e^{2 \pi i \theta}\right)=e^{2 \pi i k \theta}$ where $k$ is a non-negative integer. We have demonstrated that any continuous homomorphism $f$ of $T^{m}$ onto $T^{n}$ is isomorphic to a canonical homomorphism $q$ which is a product of $n$ homomorphisms of $T$ into $T$. That is, there are isomorphisms $r$ and $r^{\prime}$ of $T^{m}$ and $T^{n}$, respectively, such that $f=r^{\prime} \circ q \circ r$.

Let $f$ be a canonical continuous homomorphism of $T^{m}$ onto $T^{n}$ :

$$
f\left(e^{2 \pi i \theta_{1}}, \ldots, e^{2 \pi i \theta_{n}}, \ldots, e^{2 \pi i \theta_{m}}\right)=\left(e^{2 \theta i k_{1} \theta_{1}}, \ldots, e^{2 \pi i k_{n} \theta_{n}}\right) .
$$

If $m=n$, it is easily seen that $f$ is a $\prod_{i=1}^{n} k_{i}$ to one covering. In general, $f$ has connected kernel if and only if $k_{i}=1$ for each $i$ and if $A$ is a connected set of small enough diameter, then $f^{-1}(A)$ consists of $\prod_{i=1}^{n} k_{i}$ disjoint connected sets which are translates in $T^{m}$ of each other.

Suppose $G$ is a compact group and $g$ is a continuous homomorphism of $G$ onto $T^{n}$. Then $g$ preserves normalised Haar measure. This readily follows from the fact that $T^{n}$ is dyadically decomposable, that is, expressible as a disjoint union of sets which are translates of each other.

Suppose $G$ is a compact connected metrisable abelian group, then $G$ is an inverse limit space $\left\{G_{i}, p_{i}^{i+1}\right\}_{i \in \mathbb{N}}$ where the factor spaces $G_{i}=T^{n_{i}}$ for some positive integer $n_{i}$ and where the bonding maps $p_{i}^{i+1}$ are continuous surjective homomorphisms. (This wellknown result follows easily for example from Corollary 1 of Theorem 14 of [5].) We shall let $\pi_{i}$ denote the projection of $G$ onto the factor $G_{i}$, so that $\pi_{i}=p_{i}^{i+1} \circ \pi_{i+1}$. If only a finite number of the bonding maps have disconnected kernel, then $G$ can be expressed as an inverse limit space $\left\{G_{i}^{\prime}, p_{i}^{i+1}\right\}_{i \in N}$ where all the bonding maps have connected kernels. Thus by Theorem 4.3 of [1], $G$ is locally connected.

Lemma 3. Let $A$ be a closed arc contained in G. Let $\lambda$ and $\lambda_{i}$ be normalised Haar 
measures on $G$ and $G_{i}$, respectively. If $p_{i}^{i+1}$ has disconnected kernel and $\pi_{i}(A) \neq G_{i}$ then

$$
\lambda(A) \leqq \lambda_{i+1}\left(\pi_{i+1}(A)\right) \leqq \frac{1}{2} \lambda_{i}\left(\pi_{i}(A)\right)
$$

Proof. Let $\left\{A_{k}\right\}_{k}$ be a family of non-overlapping closed subarcs of $A$ such that $A$ $=\bigcup_{k} A_{k}$. Suppose the lemma holds for each $A_{k}$ separately, then the lemma holds for $A$. Therefore, we may assume without loss of generality that $A$ is of as small a diameter as we choose, so that $q_{i}^{-1}\left(\pi_{i}(A)\right)$, where $q_{i}=p_{i}^{i+1}$, is a disjoint union of closed connected sets $S_{1}, S_{2}, \ldots, S_{m}, m \geqq 2$, where each $S_{j}$ is a translate of $S_{1}$. Thus $\lambda_{i+1}\left(S_{1}\right)=\lambda_{i+1}\left(S_{j}\right)$ for each $j$ and since $p_{i}^{i+1}$ preserves Haar measure $\lambda_{i+1}\left(S_{1}\right)=(1 / m) \lambda_{j}\left(\pi_{i}(A)\right)$. By connectivity $\pi_{i+1}(A) \subseteq S_{j}$ for some $j$. This proves the lemma.

Theorem 1. Let $G$ be a compact connected metrisable abelian group. Then $G$ has the homeomorphic measure property if and only if $G$ is locally connected.

Proof. Sufficiency follows from Theorem A. Suppose $G$ is not locally connected. As $G$ contains a one parameter subgroup, $G$ contains a closed arc $S$. Let $f$ be a homeomorphism of $I$ onto $S$ and define a Borel measure $\mu$ on $G$ by $\mu(A)=m\left(f^{-1}(A \cap S)\right)$ where $m$ is linear Lebesgue measure. Define a Borel measure $\alpha$ on $G$ by $\alpha=\frac{1}{2}(\mu+\lambda)$. Then $\alpha$ is a locally positive non-atomic Borel probability measure on $G$ but cannot be homeomorphic to $\lambda$ because $\alpha(S)=\frac{1}{2}$ and by Lemma $3 \lambda(h(S))=0$ for every homeomorphism $h$ of $G$ onto itself.

Acknowledgements. We wish to thank Professor Karl Hofmann for useful discussions. The research for this paper was done while the first author was a Visiting Professor at Tulane University.

\section{REFERENCES}

1. C. E. CAPEL, Inverse limit spaces, Duke Math. 21 (1954), 233-245.

2. A. FathI, Structure of the group of homeomorphisms preserving a good measure, Annales Scientifiques de LEcole Normale Supérieure (to appear).

3. E. Hewirt and K. A. Ross, Abstract Harmonic Analysis, Vol. I, (Springer-Verlag, 1963).

4. A. B. Katok and A. B. Stepin, Metric properties of measure preserving homeomorphisms (Russian), Uspehi Mat. Nauk 25, no. 2 (152), (1970), 193-220, (Russian Mathematical Surveys 25 (1970), 191-220.

5. S. A. Morris, Pontryagin duality and the structure of locally compact abelian groups (Cambridge Univ. Press, 1977).

6. S. A. Morris and V. C. PECK, A note on the homeomorphic measure property, Colloq. Math. (to appear).

7. J. C. Oхтовy and S. M. Ulam, Measure-preserving homeomorphisms and metric transitivity, Ann. Math. (2) 42 (1941), 874-920.

8. J. C. Oxtoby and V. S. Prasad, Homeomorphic measures in the Hilbert cube, Pacific J. Math. 77 (1978), 483-497.

La TRoBe UnIVERSITY

BUNDOORA

VICTORIA 3083

Australia
TULANE UNIVERSiTY

New OrLEans

LA, 70118

U.S.A. 